\title{
The Evaluation of Liraglutide Combined with Intensive Insulin Therapy on the Pancreatic Beta Cells Function in Type II Diabetes Mellitus
}

\author{
Shenjiao Dong1, Feixia Shen ${ }^{2, *}$ \\ ${ }^{1}$ Department of Endocrinology, Taizhou Hospital Affiliated to Wenzhou Medical University, Linhai 317000, Zhejiang, China. \\ ${ }^{2}$ Department of Endocrinology, The First Affiliated Hospital of Wenzhou Medical University, Wenzhou, Zhejiang 325000, China.
}

How to cite this paper: Shenjiao Dong, Feixia Shen. (2021) On the Treatment of Children's Anorexia Based on the Theory of Spleen Yin. International Journal of Clinical and Experimental Medicine Research, 5(3), 377-382. DOI: $10.26855 /$ ijcemr.2021.07.023

Received: June 24, 2021

Accepted: July 16, 2021

Published: July 22, 2021

*Corresponding author: Feixia Shen, Department of Endocrinology, The First Affiliated Hospital of Wenzhou Medical University, Wenzhou, Zhejiang 325000, China.

\begin{abstract}
Objective: The purpose of this study was to analyze the effect of liraglutide administration combined with intensive insulin therapy on the pancreatic $\beta$ cell function and blood lipid in patients with type 2 diabetes mellitus (T2DM). Methods: The patients with T2DM admitted to our hospital from August 2017 to December 2019 were selected as study subjects. According to the treatments, a total of 200 patients were randomly divided into control group (n $=70)$ and observation group $(n=130)$. The patients in the control group were treated with glimepiride combined with intensive insulin therapy. The patients in the observation group were given liraglutide combined with intensive insulin therapy. The changes of body weight and $\beta$ cell function of patients was compared and analyzed between the two groups. Results: The values of body weight, abdominal circumference and body mass index for patients in the control group revealed no significant difference after treatment compared to non-treatment $(P>0.05)$, but these values in the observation group showed significant decline after treatment $(P<0.05)$. The two groups indicated an obvious decrease on the triglyceride (TG), Total cholesterol (TC), and Low density lipoprotein (LDL), and a significant increase on the high density lipoprotein (HDL) after treatment $(P<0.05)$. In addition, these values in the observation group were superior to that in the control group $(P<0.05)$. There was a decline on the fasting plasma glucose (FPG), glycosylated hemoglobin (HbA1c), and homeostasis model assessment-insulin resistance (HOMA-IR), and a significant increase on the homeostasis model assessment $-\beta$ (HOMA- $\beta$ ) in both groups after treatment $(P<0.05)$. But these changes were more obviously in the observation group $(P<0.05)$. Conclusion: Liraglutide administration combined with insulin intensive therapy were contributed to improving $\beta$ cell function, and reducing blood lipid and body weight.
\end{abstract}

\section{Keywords}

Liraglutide, Glimepiride, Type 2 diabetes mellitus, Insulin intensive therapy, $\beta$ cell function, Blood lipid

\section{Introduction}

Diabetes is characterized by high blood sugar and one of the biggest epidemics in the world. It involves type 1 diabetes mellitus (T1DM) and type 2 diabetes mellitus (T2DM) [1]. The T1DM is induced by the insulin deficiency, 
and the T2DM is related to the insulin resistance. The two forms are associated with the destruction of pancreatic beta cell [2]. The incidence and mortality of diabetes increased year by year, especially in China, the United States and India, in which T2DM accounted for a large proportion. The latest global estimate of the International Diabetes Federation is that there will be 642 million diabetics in 2040 [3]. In addition, the incidence of T2DM is increasing in children and teenagers [4]. The T2DM leads to a series of complications such as cardiovascular disease, kidney, retina and nervous system diseases, which can be life-threatening [5]. The evidences suggest that there is an urgent need to formulate and implement new preventive and therapeutic strategies. Since natural history of T2DM refers to a continuous process of pancreatic beta cell failure with the metabolic dysfunction, and insulin possesses anti-inflammatory and antioxidant properties. Nowadays, the insulin therapy has been remained as a basic way to control blood glucose and prevent beta cell, vascular endothelial cell, and other organs from damage caused by hyperglycemia [6, 7]. In order to enhance the efficacy, some oral antidiabetic drugs, such as metformin and sulfonylureas, have been widely applied in the treatment of T2DM [8]. However, these drugs might run the risk of weight gain, hypoglycemia, and hyperinsulinemia [9, 10]. In recent years, the treatment of T2DM with incretin therapy has been highly valued, and the relevant drugs specifically target the glucagon like peptide-1 (GLP-1) receptor and act through the incretin system [11]. The liraglutide, as a GLP-1 analogue, can increase the postprandial insulin levels through glucose dependent way, and it has been related to the weight loss and blood pressure reduction [12]. But there was little evidence indicated the liraglutide combined with intensive insulin therapy in the treatment of T2DM. The purpose of this study aimed at investigating the association between the liraglutide combined intensive insulin therapy and the blood lipid, body weight, and $\beta$ cell function for the T2DM patients.

\section{Materials and methods}

\subsection{Research subjects}

The patients with T2DM in our hospital from August 2017 to December 2019 were selected as the research subjects. A total of 200 patients were randomly divided into two groups. The control group $(n=70)$ involved 41 males and 29 females, who aged from 41-74 years, with an average of (61.4 \pm 11.5$)$ years, and the course of disease was 2-20 years, and with an average of $(13.5 \pm 8)$ year. The observation group $(n=130)$ included 78 males and 52 females, who ranged from 44-73 years, with an average of (61.9 \pm 8 ) years, and the course of disease was 2-22 years, with an average of (14.2 \pm 1$)$ years. There was no significant difference in gender ratio, average age, course of disease and other general information between the two groups $(P>0.05)$.

\subsection{Inclusion criteria and exclusion criteria}

All patients included should be met following criteria: a. Fasting plasma glucose (FPG) $\geq 7 \mathrm{mmol} /$ Lor $2 \mathrm{~h}$ postprandial plasma glucose (PPG) $\geq 11.1 \mathrm{mmol} / \mathrm{L}$; b. Age from $18-80$ years, the course of disease < 30 years; c. FPG $\geq$ $8.0 \mathrm{mmol} / \mathrm{L}$ and $2 \mathrm{~h} P P G \geq 13.9 \mathrm{mmol} / \mathrm{L}$ after 1 week of intensive insulin therapy; d. Complete clinical data and informed consent was signed voluntarily. The patients be in accord with any of following criteria should be excluded: a. Groups including end-stage renal disease, severe liver function impairment, history of pancreatitis, T1DM or diabetic ketoacidosis, pregnant and lactating women and children; b. History of medullary thyroid carcinoma or family history of tumor and blood disease; c. Non-compliant patient; d. Patients received other drugs like glucocorticoids leading to adverse reaction during study.

\subsection{Methods}

The patients in the control group were given oral glimepiride combined with intensive insulin therapy. Usually 2 mg glimepiride was taken in once a day and the dosage of glimepiride and insulin were adjusted according to the blood glucose level of patients. $6 \mathrm{mg}$ glimepiride at most per day was applied to the patients and the other oral drugs remained unchanged. On the basis of intensive insulin therapy, the patients in the observation group were received subcutaneous injection liraglutide with $0.6 \mathrm{mg}$ dosage once a day. The therapy was lasted for 12 weeks.

\subsection{Blood collection and outcome measures}

The patients were collected fasting venous blood before and 12 weeks after treatment respectively.

The blood samples were restored in a medical refrigerator at $4^{\circ} \mathrm{C}$, and centrifuged for 10 minutes at the speed of 3,000 rpm to separate the serum for testing. The FPG, low density lipoprotein (LDL), high density lipoprotein (HDL), triglyceride (TG), and total cholesterol (TC) were detected by automatic biochemical analyzer (ARCHITECT c1600, Abbott, China). The glycosylated hemoglobin (HbA1c) was examined by the glycosylated 
hemoglobin analyzer (H50, Mindray, China). The fasting insulin (FINS) was detected by the automatic electrochemiluminescence immunoassay analyzer (Cobas E601, Roche, China). All processes were in strict accordance with the instructions. The values of body weight, abdominal circumference, and body mass index were recorded, and homeostasis model assessment- $\beta$ (HOMA- $\beta$ ) $=20 \times$ FINS/(FBG-3.5), homeostasis model assessment-insulin resistance $($ HOMA-IR $)=$ FPG $\times$ FINS/22.5.

\section{Statistical analysis}

Spss20.0 statistical software was used to process the data. The measurement data were expressed by mean \pm standard deviation and analyzed by t test. The counting data were analyzed by chi-square test. $P<0.05$ was considered to be statistically significance.

\section{Results}

\subsection{The liraglutide administration decreased body weight at 12 weeks after treatment}

As listed in Table 1, the changes of the weight, abdominal circumference, and body mass index in the control group had no significant difference after treatment $(P>0.05)$, while these values in the observation group were significantly decreased $(P<0.05)$.

\subsection{The liraglutide administration was quite effective in adjusting blood lipid at 12 weeks after treat- ment}

As showed in the Table 2, the values of TG, TC and LDL were significantly decreased, and HDL obviously increased in the two groups after treatment $(P<0.05)$. But these values in the observation group were superior to those in the control group $(P<0.05)$.

Table 1. Changes of body weight, abdominal circumference and body mass index between the two groups

\begin{tabular}{ccccc}
\hline & Group & Body weight $(\mathrm{kg})$ & Body mass index $\left(\mathrm{kg} / \mathrm{m}^{2}\right)$ & Abdominal circumference $(\mathrm{cm})$ \\
\hline \multirow{2}{*}{ Control group } & Before treatment & $85.6 \pm 10.2$ & $29.3 \pm 4.5$ & $103.5 \pm 11.2$ \\
& At 12 weeks after treatment & $84.6 \pm 8.3$ & $29.1 \pm 5.8$ & $102.3 \pm 10.8$ \\
$\mathrm{t}$ & & 0.754 & 0.258 & 0.347 \\
$\mathrm{P}$ & & $>0.05$ & $>0.05$ & $>0.05$ \\
& Before treatment & $85.8 \pm 8.7$ & $29.5 \pm 6.1$ & $103.6 \pm 11.7$ \\
Observation group & At 12 weeks after treatment & $80.4 \pm 9.2 *$ & $26.4 \pm 5.7 *$ & $95.9 \pm 9.6^{*}$ \\
$\mathrm{t}$ & & 4.637 & 5.285 & 4.636 \\
$\mathrm{P}$ & & $<0.05$ & $<0.05$ & $<0.05$ \\
\hline
\end{tabular}

The sign of * indicated $P<0.05$ compared with control group.

Table 2. The blood lipid changes between the two groups

\begin{tabular}{cccccc}
\hline & Group & TG $(\mathrm{mmol} / \mathrm{L})$ & TC $(\mathrm{mmol} / \mathrm{L})$ & LDL $(\mathrm{mmol} / \mathrm{L})$ & HDL $(\mathrm{mmol} / \mathrm{L})$ \\
\hline \multirow{2}{*}{ Control group } & Before treatment & $2.69 \pm 0.54$ & $5.93 \pm 0.92$ & $3.76 \pm 0.78$ & $1.21 \pm 0.42$ \\
& At 12 weeks after treatment & $2.15 \pm 0.43$ & $4.23 \pm 0.87$ & $3.03 \pm 0.69$ & $1.47 \pm 0.60$ \\
$\mathrm{t}$ & & 4.275 & 5.128 & 4.158 & 8.052 \\
$\mathrm{P}$ & & $<0.05$ & $<0.05$ & $<0.05$ & $<0.05$ \\
& & $2.671 \pm 0.78$ & $5.98 \pm 0.74$ & $3.80 \pm 0.99$ & $1.18 \pm 0.55$ \\
Observation group & Before treatment & $1.62 \pm 0.54^{*}$ & $2.90 \pm 0.82^{*}$ & $2.08 \pm 0.72^{*}$ & $1.81 \pm 0.68^{*}$ \\
$\mathrm{H}$ & At 12 weeks after treatment & 3.978 & 4.441 & 3.879 & 6.315 \\
$\mathrm{P}$ & & $<0.05$ & $<0.05$ & $<0.05$ & $<0.05$ \\
\hline
\end{tabular}

The sign of * indicated $P<0.05$ compared with control group.

TG: triglyceride; TC: total cholesterol; LDL: low density lipoprotein; HDL: high density lipoprotein. 


\subsection{The liraglutide administration reduced blood glucose and improved $\beta$ cell function at 12 weeks after treatment}

It was found that the FPG, HbA1c, and HOMA-IR value were significantly decreased, and HOMA- $\beta$ value was obviously increased in the control group and in the observation group after treatment $(P<0.05)$. The changes of the above indexes in the observation group were more obvious compared to the control group ( $P<0.05$, see Table 3 ).

Table 3. Comparison of blood glucose, insulin resistance and $\beta$ cell function between the two groups

\begin{tabular}{|c|c|c|c|c|c|}
\hline & Group & $\mathrm{FPG}(\mathrm{mmol} / \mathrm{L})$ & HbA1c (\%) & HOMA- $\beta$ & HOMA-IR \\
\hline \multirow{2}{*}{ Control group } & Before treatment & $9.88 \pm 1.67$ & $10.3 \pm 2.04$ & $34.51 \pm 5.98$ & $3.20 \pm 1.36$ \\
\hline & At 12 weeks after treatment & $7.31 \pm 0.87$ & $8.9 \pm 0.8)$ & $38.18 \pm 6.34$ & $2.45 \pm 0.85$ \\
\hline $\mathrm{t}$ & & 3.658 & 4.206 & 4.856 & 5.361 \\
\hline $\mathrm{p}$ & & $<0.05$ & $<0.05$ & $<0.05$ & $<0.05$ \\
\hline \multirow{2}{*}{ Observation group } & Before treatment & $9.97 \pm 1.47$ & $10.4 \pm 2.23$ & $34.78 \pm 6.99$ & $3.30 \pm 1.48$ \\
\hline & At 12 weeks after treatment & $6.56 \pm 0.65^{*}$ & $8.5 \pm 1.7^{*}$ & $51.36 \pm 7.38 *$ & $1.92 \pm 0.61^{*}$ \\
\hline $\mathrm{t}$ & & 3.639 & 3.503 & 4.086 & 5.648 \\
\hline $\mathrm{P}$ & & $<0.05$ & $<0.05$ & $<0.05$ & $<0.05$ \\
\hline
\end{tabular}

FPG: fasting plasma glucose; HbA1c: glycosylated hemoglobin; HOMA- $\beta$ : homeostasis model assessment- $\beta$; HOMA-IR: homeostasis model assessment-insulin resistance.

\section{Discussion}

T2DM is one of the main diseases that affect people's health and survival in modern society, and its complications are associated with the main clinical death [5]. The T2DM represents insulin resistance, and the insulin therapy is an important way to improve insulin resistance [13]. In the past, the treatment of T2DM is mainly based on diet control, an oral hypoglycemic drug or combined with insulin threapy. But the main target cell of this treatment is $\beta$ cell, unable to act on $\alpha$ cell [14]. At the same time, with the increase of long-term drug dosage and type, patients may have adverse reactions such as hypoglycemia, obesity and cardiovascular events [8]. Therefore, in order to avoid the above adverse reactions, the treatment on targeting the $\beta$ cell and $\alpha$ cell is in necessity. The liraglutide has been proved to protect $\beta$ cell and $\alpha$ cell, which leaded to control blood sugar [15].

The purpose of this study was to analyze the effect of liraglutide combined with intensive insulin therapy on patients with T2DM. According to the data, there were no significant changes in body weight, abdominal circumference and body mass index in the control group after treatment, but these values in the observation group were significantly decreased. These findings indicated that liraglutide administration promoted weight loss. It was found that the administration of semaglutide and liraglutide significantly reduced the body weight of obese patients compared to placebo [16]. The study has indicated that compared with exenatide and sitagliptin, long-term use of liraglutide combined with metformin in T2DM patients revealed longer life expectancy [17]. In addition, this study indicated that the values of TG, TC and LDL were significantly decreased, and HDL obviously increased in the two groups after treatment, but these changes in the observation group were superior to those in the control group. The values showed that the liraglutide administration could regulate blood lipid for T2DM patients. Some researches indicated short-term use of high dose liraglutide can reduce the concentration of total cholesterol, free cholesterol, residual cholesterol which related to the cardiovascular disease [18], and the TC, TG, and LDL values of mice were significantly reduced by the liraglutide administration, also the liver lipid deposition in HFD fed dbb/db mice was reduced [19]. Some studies about the mouse model of T2DM suggested that liraglutide enhanced autophagy and promoted the proliferation of pancreatic function $\beta$ cell [20]. The T2DM patients' blood glucose can be stabilized by using liraglutide on the basis of the metformin treatment, which can significantly reduce the level of HbA1c and increase weight loss [21]. In this analysis, it was found that the FPG, HbA1c, and HOMA-IR value were significantly decreased, and HOMA- $\beta$ value was obviously increased in the control group and in the observation group after treatment, but these changes in the observation group were more obvious. It suggested that liraglutide combined with intensive insulin therapy is effective treatment for patients with T2DM, which controlled the blood glu- 
cose and improved pancreatic $\beta$ cell function. In the future study, we will further verify this hypothesis by establishing an animal model of T2DM, and analyze the pathological changes and recovery of the key parts in the pancreatic pathological section. At the same time, we will also use agonist and inhibitor methods to verify the correctness of the conclusion.

Therefore, liraglutide combined with intensive insulin therapy is an effective way for patients with T2DM, which improves the $\beta$ cell function, regulates blood lipid and reduces the weight of patients.

\section{References}

[1] A. M. Schmidt. (2018). "Highlighting Diabetes Mellitus: The Epidemic Continues.” Arterioscler Thromb Vasc Biol., vol. 38, no. 1, pp. e1-e8, 2018.

[2] A. American Diabetes. (2015). “(2) Classification and diagnosis of diabetes.” Diabetes Care, vol. 38, Suppl, pp. S8-S16, 2015.

[3] P. Zimmet, K. G. Alberti, D. J. Magliano, and P. H. Bennett. (2016). "Diabetes mellitus statistics on prevalence and mortality: facts and fallacies.” Nat Rev Endocrinol, vol. 12, no. 10, pp. 616-622, 2016.

[4] K. T. Kao and M. A. Sabin. (2016). “Type 2 diabetes mellitus in children and adolescents.” Aust Fam Physician, vol. 45, no. 6, pp. 401-406, 2016.

[5] Y. Zheng, S. H. Ley, and F. B. Hu. (2018). "Global aetiology and epidemiology of type 2 diabetes mellitus and its complications.” Nat Rev Endocrinol, vol. 14, no. 2, pp. 88-98, 2018.

[6] M. Hanefeld, H. Fleischmann, T. Siegmund, and J. Seufert. (2020). "Rationale for Timely Insulin Therapy in Type 2 Diabetes Within the Framework of Individualised Treatment: 2020 Update.” Diabetes Ther, vol. 11, no. 8, pp. 1645-1666, 2020.

[7] D. R. Owens. (2013). “Clinical evidence for the earlier initiation of insulin therapy in type 2 diabetes.” Diabetes Technol Ther, vol. 15, no. 9, pp. 776-785, 2013.

[8] S. E. Inzucchi, R. M. Bergenstal, J. B. Buse, M. Diamant, E. Ferrannini, M. Nauck, A. L. Peters, A. Tsapas, R. Wender, and D. R. Matthews. (2012). "Management of hyperglycaemia in type 2 diabetes: a patient-centered approach. Position statement of the American Diabetes Association (ADA) and the European Association for the Study of Diabetes (EASD).” Diabetologia, vol. 55, no. 6, pp. 1577-1596, 2012.

[9] C. L. Morgan, C. D. Poole, M. Evans, A. H. Barnett, S. Jenkins-Jones, and C. J. Currie. (2012). "What next after metformin? A retrospective evaluation of the outcome of second-line, glucose-lowering therapies in people with type 2 diabetes.” $J$ Clin Endocrinol Metab, vol. 97, no. 12, pp. 4605-4612, 2012.

[10] M. Ridderstrale, K. R. Andersen, C. Zeller, G. Kim, H. J. Woerle, U. C. Broedl, and E.-R. H. H. S. T. Investigators. (2014). "Comparison of empagliflozin and glimepiride as add-on to metformin in patients with type 2 diabetes: a 104-week randomised, active-controlled, double-blind, phase 3 trial.” Lancet Diabetes Endocrinol, vol. 2, no. 9, pp. 691-700, 2014.

[11] M. Nauck. (2016). "Incretin therapies: highlighting common features and differences in the modes of action of glucagon-like peptide-1 receptor agonists and dipeptidyl peptidase-4 inhibitors.” Diabetes Obes Metab, vol. 18, no. 3, pp. 203-216, 2016.

[12] S. P. Marso, G. H. Daniels, K. Brown-Frandsen, P. Kristensen, J. F. Mann, M. A. Nauck, S. E. Nissen, S. Pocock, N. R. Poulter, L. S. Ravn, W. M. Steinberg, M. Stockner, B. Zinman, R. M. Bergenstal, J. B. Buse, L. S. Committee, and L. T. Investigators. (2016). "Liraglutide and Cardiovascular Outcomes in Type 2 Diabetes.” N Engl J Med., vol. 375, no. 4, pp. 311-322, 2016.

[13] N. Rachdaoui. (2020). "Insulin: The Friend and the Foe in the Development of Type 2 Diabetes Mellitus.” Int J Mol Sci., vol. 21, no. 5, 2020.

[14] F. A. M. Al-Omary. (2017). “Gliclazide.” Profiles Drug Subst Excip Relat Methodol, vol. 42, pp. 125-192, 2017.

[15] W. A. Nuffer and J. M. Trujillo. (2015). “Liraglutide: A New Option for the Treatment of Obesity.” Pharmacotherapy, vol. 35, no. 10, pp. 926-934, 2015.

[16] P. M. O'Neil, A. L. Birkenfeld, B. McGowan, O. Mosenzon, S. D. Pedersen, S. Wharton, C. G. Carson, C. H. Jepsen, M. Kabisch, and J. P. H. Wilding. (2018). "Efficacy and safety of semaglutide compared with liraglutide and placebo for weight loss in patients with obesity: a randomised, double-blind, placebo and active controlled, dose-ranging, phase 2 trial.” Lancet, vol. 392, no. 10148, pp. 637-649, 2018.

[17] C. Tzanetakos, A. Melidonis, C. Verras, G. Kourlaba, and N. Maniadakis. "Cost-Effectiveness Analysis of Liraglutide Versus Sitagliptin or Exenatide in Patients With Inadequately Controlled Type 2 Diabetes On Oral Antidiabetic Drugs In Greece.” Value Health, vol. 17, no. 7, p. A345, 2014.

[18] N. Peradze, O. M. Farr, N. Perakakis, I. Lazaro, A. Sala-Vila, and C. S. Mantzoros. (2019). "Short-term treatment with high dose liraglutide improves lipid and lipoprotein profile and changes hormonal mediators of lipid metabolism in obese patients with no overt type 2 diabetes mellitus: a randomized, placebo-controlled, cross-over, double-blind clinical trial.” Cardiovasc Diabetol, vol. 18, no. 1, p. 141, 2019.

[19] Y. R. Wu, X. Y. Shi, C. Y. Ma, Y. Zhang, R. X. Xu, and J. J. Li. (2019). "Liraglutide improves lipid metabolism by enhancing cholesterol efflux associated with ABCA1 and ERK1/2 pathway.” Cardiovasc Diabetol, vol. 18, no. 1, pp. 146, 2019. 
[20] M. Fan, H. Jiang, Y. Zhang, Y. Ma, L. Li, and J. Wu. (2018). "Liraglutide Enhances Autophagy and Promotes Pancreatic beta Cell Proliferation to Ameliorate Type 2 Diabetes in High-Fat-Fed and Streptozotocin-Treated Mice.” Med Sci Monit, vol. 24, pp. 2310-2316, 2018.

[21] M. Li, Y. Yang, D. Jiang, M. Ying, Y. Wang, and R. Zhao. (2017). "Efficacy and safety of liraglutide versus sitagliptin both in combination with metformin in patients with type 2 diabetes: A systematic review and meta-analysis.” Medicine (Baltimore), vol. 96, no. 39, p. e8161, 2017. 